\title{
Dinámicas comunitarias y acceso al sistema sanitario: una participación desigual
}

\author{
Ana Isabel Gil Lacruz \\ Universidad Autónoma de Madrid \\ ana.gil@uam.es \\ Marta Gil Lacruz \\ Universidad de Zaragoza \\ mglacruz@unizar.es
}

\section{Resumen}

Las desigualdades sociales condicionan tanto la participación comunitaria como el acceso y la correcta utilización de los recursos sociales, como los sanitarios. En este sentido, la población femenina puede estar sufriendo una doble discriminación. En esta investigación nos planteamos dos objetivos: en primer lugar, analizar desde una configuración territorial y comunitaria concreta las dinámicas de acceso al servicio sanitario y la respuesta diferencial de dichos recursos; en segundo lugar, examinar la percepción e integración comunitaria de la población en función del sexo.

Palabras clave: salud comunitaria, apoyo, integración, recursos sanitarios, género.

\begin{abstract}
Social inequalities influence community participation as well as the use of social resources such as bealth care. In this respect, women could be experiencing double discrimination. Our research has two aims: first, to analyze health care access in a particular region and community and the differing provision of these resources; second, to study how people integrate into and perceive the community as a function of gender.
\end{abstract}

Key words: Community Health, Social Support, Integration, Health Care Resources, Gender. 


\section{Introducción}

Este trabajo sobre la salud comunitaria del barrio de Casablanca se inicia en 1996 gracias a la subvención concedida por el Fondo de Investigación Sanitaria del Ministerio de Sanidad y Consumo (96-0291). En esa primera fase se configuró el equipo interdisciplinar: trabajadores sociales, profesores de primaria, sociólogos, psicólogos y médicos, que se ocuparán de elaborar e implantar la Encuesta de Salud del distrito, en la que se basan nuestros datos.

Posteriormente, el Gobierno de Aragón, CONSI + D (P14/98), facilitó los recursos económicos necesarios para materializar la segunda fase del estudio, en la que, dentro de esa línea de análisis psicosocial, se trabajó fundamentalmente con las preguntas relativas a los comportamientos sanitarios de la encuesta. A partir de 2003, el Instituto de la Mujer I+D+I facilitó, con su apoyo económico, la lectura de género de la investigación, que presentamos a continuación (exp. 35/03. Título del proyecto de investigación: «Mujer y salud: aproximación psicosocial y comunitaria»).

El sexo sigue constituyendo un factor relevante de discriminación social en nuestra sociedad actual, una discriminación que se extiende al propio proceso de investigación científica sobre la salud y que se traduce en carencias metodológicas en su estudio. Se siguen utilizando instrumentos de evaluación de la salud física y mental sesgados en su diseño y que no permiten evaluar la salud en función del sexo, ni profundizar en los parámetros que contribuyen a un adecuado estado de salud.

Estas desigualdades reproducen distintas estructuras de poder, prestigio, estatus, respeto y autoestima que conllevan una mayor vulnerabilidad. A esto se suma el impacto de las condiciones de vida en tanto que generadoras de desigualdades en la salud (Arias et alii, 1993; Armero et alii, 1991).

La concepción actual de lo que es la salud se centra en sus dimensiones físicas, mentales y sociales, y caracteriza, ya no sólo al individuo, sino también a las comunidades (Gordon et alii, 1999; Hahn et alii, 1999; OMS, 1986, 1996¹).

La participación como indicador social de la salud se entiende como «el proceso en virtud del cual los individuos y las familias asumen responsabilidades en cuanto a la salud y el bienestar propios y de la colectividad; mejoran la capacidad de contribuir a su desarrollo económico y al comunitario, pues al ser los mejores conocedores de su propia situación son capaces de encontrar incentivos que resuelven sus problemas

\footnotetext{
1 De hecho, la Organización Mundial de la Salud ha creado una comisión específica para el estudio de los determinantes sociales de la salud. En su página web (http:// who.int/social_determinants) se facilita abundante evidencia empírica sobre el impacto de estas dimensiones en la salud. Recomendamos, como ejemplo, el texto de Richard Wilkinson y Michael Marmot (2004): Social determinants of healtb: the solid facts. 2 nd edition. WHO Library Cataloguing in Publication Data.
} 
comunes». Esto les permite ser agentes de su propio desarrollo y, al mismo tiempo, beneficiarios reales de la ayuda al cambio.

La participación se entiende, por tanto, como una expresión de responsabilidad social: es un derecho y un deber de todo ciudadano. En materia de salud, abarca todos los proyectos y actividades que afectan a la propia salud y, en concreto, a la planificación o a la gestión de la salud comunitaria, expresando sus necesidades y colaborando en su priorización, en la solución de sus problemas y en la planificación de los servicios sanitarios (Martínez et alii, 1996).

La participación de las mujeres en el desarrollo de su salud es un requisito básico para el bienestar comunitario. Las políticas y los programas institucionales empiezan a reconocer su opinión en los procesos de identificación y planificación del sistema sanitario (Duro et alii, 2001; Ministerio de Sanidad y Consumo, 2003²). Hasta ahora, los procesos relativos a la participación y el desarrollo socioeconómico no han afectado en la misma medida a hombres y mujeres. La perspectiva de género puede contribuir a modificar el concepto sesgado de desarrollo y a tener en cuenta también el logro de una mayor igualdad entre los ciudadanos (Yago et alii, 2002).

La investigación sociosanitaria, además de promover la igualdad entre hombres y mujeres, debe profundizar en la interacción del sexo con otros factores de diferenciación y estratificación social, como la etnia, la edad, el nivel de ingresos, la religión, la orientación sexual, etc. (Esteban, 199633 Navarro et alii, 1994; Olson y Shultz, 1994).

Por ejemplo, para ambos sexos, el uso de la consulta médica está mediatizado por variables como la edad, el nivel de estudios, los ingresos mensuales familiares o la clase social. Además, hombres y mujeres, conforme tienen más años, consultan más al médico, consumen más medicamentos y, excluido el motivo del parto, se hospitalizan más. Así, en nuestro país, más de la mitad de nuestros jóvenes (56\%) se considera totalmente sano, mientras que ese porcentaje va descendiendo con la edad hasta el $11 \%$ en las personas mayores de 60 años (De Miguel, 1997²). Ahora bien, a igualdad de años en nuestras encuestas nacionales de salud, las mujeres afirman sentirse peor que los varones.

\footnotetext{
2 El Ministerio de Sanidad y Consumo sostiene que «para conseguir los más altos niveles de salud, las políticas sanitarias deben reconocer que las mujeres y los hombres, debido a diferencias biológicas y a sus roles de género, tienen diferentes necesidades, obstáculos y oportunidades».

3 Los textos de la profesora Esteban constituyen un buen ejemplo para el estudio de las diferencias de sexo en relación con el cuerpo. Entre otros libros de referencia que profundizan en aspectos concretos de esta desigualdad destacamos los siguientes: Esteban, M. L. (2001). Re-producción del cuerpo femenino. Discursos y prácticas acerca de la salud. San Sebastián: Gakoa. Esteban, M. L. (2004). Antropología del cuerpo. Género, itinerarios corporales, identidad y cambio. Bellaterra: Barcelona.

4 La investigación que aquí citamos del profesor De Miguel completa el ciclo de informes anuales que se inició en 1992 bajo el auspicio de la Fundación General de la Universidad Complutense. Se basa en encuestas anuales (de unas 5.000 entrevistas cada una). En este volumen, el análisis se centra en el terreno de los valores, sentimientos y afectos. La salud no es ajena a esta doble vertiente de funcionamiento objetivo y estado percibido.
} 
Se podría matizar que las mujeres presentan más achaques de salud a partir de los 45 años. Hasta esa edad, los porcentajes de salud deficiente están más igualados. Es lógico que la diferencia se amplíe en los mayores de 65 años, porque este grupo comprende más mujeres que varones.

Advertimos una tendencia similar al relacionar las variables sexo y edad con el estado civil. Entre las personas solteras, sólo un 5\% se siente enfermas; para las separadas, ese porcentaje alcanza el $13 \%$; para las casadas, un $17 \%$; y para las viudas, un $42 \%$. Las personas que manifiestan sentirse mejor son aquellos jóvenes que viven con sus padres, pues sólo un 3\% afirma sentirse enfermo. Las personas mayores que viven solas son las que peor se encuentran (12\%). En este grupo se integra el colectivo de mujeres viudas (De Miguel, 1997).

Si mantenemos constante el sexo y la edad, según desciende el nivel socioeconómico aumenta la proporción de quienes manifiestan sentirse peor. El número de consultas médicas disminuye conforme aumenta el nivel de estudios, bien sea el propio, bien sea el del cabeza de familia. De igual modo se puede interpretar la tendencia inversa: la educación atenúa el efecto de la edad en las mujeres con relación al uso de la consulta médica, del consumo de medicamentos y de la visita al dentista (Alonso y Antó, 1986; Duran, 1983, Gil Lacruz, 20045 ). También para ambos sexos, la frecuencia de consultas disminuye conforme aumentan los ingresos mensuales familiares.

Si entendemos por clase social el conjunto de personas que comparten similares condiciones de vida en materia de educación, ingresos económicos y situación laboral, observamos que el hecho de ser mujer añade un elemento distintivo a los procesos de desigualdad social. El sexo hace más profundas las diferencias, manteniendo constante la clase social. Así, por ejemplo, en las mujeres ancianas, la riqueza se relaciona con una utilización más frecuente de lo servicios médicos.

Las mujeres que trabajan fuera de casa, respecto a los hombres en igual situación laboral, frecuentan en mayor medida las consultas médicas y del dentista y consumen más medicamentos. En cuanto a las diferencias entre mujeres, las que trabajan en casa visitan menos al dentista y acuden más a las urgencias que las mujeres que trabajan fuera del hogar, independientemente de su edad (Clark, 1990).

En nuestro entorno, en el que conviven dos sistemas sanitarios, el público y el privado, la clase acomodada disfruta de una mejor atención sanitaria o, cuando menos, de la posibilidad de elegir (De Miguel, 1997). Las personas precarizadas utilizan fundamentalmente la sanidad pública, mientras que, cuanto mejor es la posición social, se acude más a los seguros privados y, sobre todo, a la combinación de ambos sistemas (Cais y Castilla, 1995).

5 La investigadora Ana Isabel Gil Lacruz presentó en 2004 una interesante tesis doctoral sobre el impacto del capital humano y social en los comportamientos saludables y de riesgo. Para ello, utilizó como bases de datos la Encuesta sobre Drogas a la Población Escolar 2000 (compuesta de una muestra de 17.168 individuos). 
Este panorama se agrava en el caso de las mujeres inmigrantes. Yago (2002) indica que la inmigración femenina conlleva un mayor grado de sufrimiento y de discriminación en temas de salud, ya que a las dificultades del proceso migratorio se sumarían las propias de ser mujeres en un marco cultural diferente y sin los recursos propios de su red social.

Necesitamos políticas sociales que engloben acciones locales en el área de la cultura, la educación, el trabajo, la salud, el consumo, el medio ambiente, etc., dentro del marco de planes integrales dirigidos a los diferentes colectivos que integran la comunidad. Con la transversalidad efectiva de la salud, se consigue optimizar el uso de los recursos, generar un trabajo de mayor calidad y dinamizar la participación, promoviendo mecanismos de solidaridad (Moreno y Pérez, 1992).

\section{Material y métodos}

El universo de la presente investigación se compone de 6.514 personas mayores de 14 años y empadronadas en el barrio zaragozano de Casablanca (3.136 hombres y 3.378 mujeres). La elección de dicho universo se realizó a partir de criterios técnicos y sociales. Casablanca constituye una comunidad marcada por los contrastes y las diferencias de composición sociodemográfica (Federación de Asociaciones de Vecinos. Zaragoza, 1981). La estratificación social se proyecta claramente en las tres subzonas de residencia: Viñedo Viejo, el núcleo originario, hoy precarizado; Fuentes Claras, con urbanizaciones de nivel socioeconómico elevado; y Las Nieves, habitada por clase media y obrera).

A partir de los datos del Padrón Municipal, la población se dividió en grupos o estratos y a continuación se obtuvo una muestra proporcional representativa. Los dos criterios básicos de estratificación de la muestra fueron la edad (jóvenes de 15 a 29 años, adultos de 30 a 59 y tercera edad, mayores de 60 años) y el sexo (hombre y mujer).

Como se trabaja con una población finita (menos de 100.000 elementos y $\mathrm{N}<$ 20 n, la población contiene menos de 20 veces el tamaño muestral (Mateo y García, 1989). Para que la muestra sea representativa a un nivel de confianza de $95,5 \%$ y con un margen de error de \pm 3 (en el caso $\mathrm{p} / \mathrm{q}=50 / 50$ ), contempla 938 encuestas individuales (más un 10\% de reserva: 94 encuestas). En total, se ha entrevistado a 452 hombres y a 486 mujeres del barrio. 
Tabla 1. Universo y muestra de la población de Casablanca

\begin{tabular}{|c|c|c|c|c|c|c|}
\hline \multirow{3}{*}{$\begin{array}{l}\text { Intervalos en } \\
\text { función de la } \\
\text { edad / sexo }\end{array}$} & \multicolumn{4}{|c|}{ Población de Casablanca $(\mathrm{N}=6.514)$} & \multicolumn{2}{|c|}{ Muestra $(\mathrm{N}=938)$} \\
\hline & \multicolumn{2}{|c|}{ Hombres } & \multicolumn{2}{|c|}{ Mujeres } & \multirow{2}{*}{$\begin{array}{c}\text { Hombres } \\
\mathrm{N}^{\circ} \\
\text { cuestionarios }\end{array}$} & \multirow{2}{*}{$\begin{array}{c}\text { Mujeres } \\
\mathrm{N}^{\circ} \\
\text { cuestionarios }\end{array}$} \\
\hline & $\begin{array}{c}\text { Fr. } \\
\text { absoluta }\end{array}$ & $\begin{array}{c}\text { Fr. } \\
\text { relativa }\end{array}$ & $\begin{array}{c}\text { Fr. } \\
\text { absoluta }\end{array}$ & $\begin{array}{c}\text { Fr. } \\
\text { relativa }\end{array}$ & & \\
\hline De 15 a 19 años & 410 & 6,29 & 412 & 6,32 & 59 & 59 \\
\hline De 20 a 24 años & 371 & 5,70 & 359 & 5,51 & 53 & 52 \\
\hline De 25 a 29 años & 312 & 4,79 & 265 & 4,07 & 45 & 38 \\
\hline Total juventud & 1.093 & 16,78 & 1.036 & 15,90 & 157 & 149 \\
\hline De 30 a 34 años & 225 & 3,45 & 231 & 3,55 & 32 & 33 \\
\hline De 35 a 39 años & 223 & 3,42 & 252 & 3,87 & 32 & 36 \\
\hline De 40 a 44 años & 275 & 4,22 & 327 & 5,02 & 40 & 47 \\
\hline De 45 a 49 años & 289 & 4,44 & 319 & 4,90 & 42 & 46 \\
\hline De 50 a 54 años & 260 & 3,99 & 231 & 3,55 & 37 & 33 \\
\hline De 55 a 59 años & 224 & 3,44 & 247 & 3,79 & 32 & 36 \\
\hline Total adultos & 1.496 & 22,97 & 1.607 & 24,67 & 215 & 231 \\
\hline De 60 a 64 años & 196 & 3,01 & 196 & 3,01 & 28 & 28 \\
\hline De 65 a 69 años & 137 & 2,10 & 199 & 3,05 & 20 & 29 \\
\hline De 70 a 74 años & 102 & 1,57 & 110 & 1,69 & 15 & 16 \\
\hline De 75 a 79 años & 55 & 0,84 & 101 & 1,55 & 8 & 15 \\
\hline$>$ de 80 años & 57 & 0,88 & 129 & 1,98 & 8 & 19 \\
\hline Total tercera edad & 547 & 8,40 & 735 & 11,28 & 79 & 106 \\
\hline Totales & 3.136 & 48,14 & 3.378 & 51,86 & 452 & 486 \\
\hline
\end{tabular}

Como pretendemos profundizar en la definición de bienestar, hemos contemplado en el diseño de la encuesta, entre otros, los siguientes indicadores:

1. Características sociodemográficas. El primer test de la encuesta consta de indicadores de estratificación social (situación laboral, nivel de estudios, ocupación, ingresos anuales, etc.), territoriales (lugar de nacimiento, lugar de residencia, años de residencia, etc.) y familiares (estado civil, número de miembros que residen en la unidad familiar, parentesco, etc.).

2. Comportamientos y recursos sanitarios. El instrumento específico sobre los recursos formales de salud en el distrito (Centro de Salud - Casablanca) contempla las siguientes dimensiones: utilización de servicios sanitarios, consultas médicas y de trabajo social; motivo; profesional; tiempo de espera y consulta; satisfacción con la atención recibida; utilización de servicios privados y/o alternativos (curanderos, homeópatas, naturistas, acupuntores, otros); y, a propósito de estos últimos, motivo, tiempo de espera y consulta, y satisfacción con la atención recibida.

3. Dinámicas de apoyo e integración comunitarias. La escala Apoyo Comunitario -90 (AC-90 de Gracia y Musitu, 1990) evalúa con un formato de cuestionario aspec- 
tos estructurales de la red de relaciones sociales de la persona, enmarcados en cuatro subescalas que permiten evaluar el apoyo social comunitario en términos de grado de integración social, participación y pertenencia a organizaciones y grupos formales e informales y utilización de servicios y recursos institucionales de la comunidad. Consúltese la tabla 2 y el cuestionario anexo para analizar la operacionalización de estas variables.

También recoge aspectos cualitativos de las relaciones que se establecen en la comunidad, tales como el grado de satisfacción y las actitudes y sentimientos hacia el vecindario y la comunidad. El coeficiente alfa de fiabilidad para el total de los ítems de la escala es elevado $(\alpha=0,90)$.

Tabla 2. Operativización de las variables de la escala de apoyo e integración comunitaria

\begin{tabular}{|lll|}
\hline Variable & Nombre & Elementos \\
\hline PartF1 & Participación & $06+07+08+10+(12-(09+11))$ \\
PartF2 & Integración & $02+03+05+(12-(01+04))$ \\
PartF3 & Sistemas informales & $12+14+17+18+19+20+(24-(13+15+16+21))$ \\
PartF4 & Sistemas formales & $22+24+25+(6-(23))$ \\
\hline
\end{tabular}

\section{Resultados}

\subsection{Características sociodemográficas}

El promedio de edad de los encuestados es de 41 años. Conforme aumenta la edad, encontramos un mayor número de mujeres. Es preciso destacar así mismo que la distribución de sexos y de edades difiere en función de la zona de residencia, ya que es en Viñedo Viejo donde encontramos una mayor presencia de mujeres mayores.

Estas diferencias se reflejan en el estado civil, ya que de nuevo, en el núcleo originario del barrio, encontramos una mayor proporción de matrimonios y personas viudas. En cambio, los solteros y solteras se asientan en las zonas de reciente colonización (Fuentes Claras).

En la muestra seleccionada trabaja un $36,9 \%$ de los encuestados. Sin embargo, esta cifra oculta importantes diferencias territoriales. Mientras que en Fuentes Claras este porcentaje es del 48,1\%, en Viñedo Viejo es del 35\%. En Fuentes Claras, la proporción de personas que se dedican a tareas del hogar $(11,5 \%)$ también es sensiblemente inferior que en Viñedo Viejo (24,9\%). 
Tres de cada cuatro personas entrevistadas $(75,8 \%)$ han cursado la enseñanza elemental y/o secundaria. Un 5,3\% de la muestra no ha estudiado y un 18,9\% ha alcanzado una titulación universitaria. Parte de esta diversidad se proyecta en el nivel de ingresos: un 47,8\% de los encuestados obtiene unos ingresos anuales inferiores a los dos millones de pesetas; sólo un $15,1 \%$ supera los cuatro millones.

Respecto al tiempo de residencia de los vecinos en el barrio, observamos cómo más de la mitad de los encuestados de Fuentes Claras (59,6\%), de manera coherente con su reciente ubicación, residen en la comunidad desde hace menos de 9 años. Por el contrario, en Viñedo Viejo una cifra similar $(59,1 \%)$ se corresponde con los que viven en el lugar desde hace más de 20 años.

\subsection{Comportamientos y recursos sanitarios}

A pesar de que el $65 \%$ de la muestra afirma haber padecido algún tipo de dolencia, sólo el $30 \%$ de los encuestados ha recurrido a los recursos sanitarios durante las dos semanas anteriores a la entrevista. La tendencia es acudir en primera instancia a servicios sanitarios de titularidad pública. El Centro de Salud de Casablanca (35,4\%), otros centros de salud (17\%) y el ambulatorio de la Seguridad Social (especialidades: $12,9 \%$ ) constituyen los principales recursos de asistencia; con menos frecuencia encontramos las consultas privadas (12,2\% particular, mutua privada). Además de una mayor utilización de los servicios sanitarios por parte de las mujeres del barrio, observamos también cómo el $81,8 \%$ de las visitas realizadas a la trabajadora social del centro de salud corresponden a mujeres, mientras que sólo un $14,3 \%$ de las mujeres del barrio han acudido a los servicios sanitarios del lugar de trabajo.

El principal motivo de consulta es el relativo al diagnóstico o tratamiento de dolencias y enfermedades. Sólo un 10\% de los encuestados ha acudido a hacerse una revisión, frente al 34,6\% que lo ha hecho para que se le dispensen recetas. El que una de cada tres consultas se haya realizado con este último fin contribuye a aumentar el grado de medicalización de la población de referencia. De hecho, al analizar la prescripción de fármacos como motivo de la consulta, el 56,3\% de las respuestas corresponde a las mujeres. La prescripción de fármacos como solución de la consulta se reserva en un $60 \%$ para las mujeres.

La revisión y los partes de alta y baja constituyen los siguientes motivos por orden de importancia (10,4\%). Si sumamos los motivos burocráticos (dispensa de recetas, revisión y parte de alta y baja), éstos explican casi la mitad de las consultas realizadas $(47,6 \%)$. 
En cuanto a la duración de la consulta, un 70,5\% de las entrevistas del barrio ha durado más de 10 minutos. La media de la visita es de 15 minutos y la puntuación más frecuente es de 10. Las mujeres esperan más tiempo que sus compañeros, pero la duración de sus consultas es mayor. El promedio de espera de los vecinos de Casablanca es de 18 minutos; sin embargo, esta cifra depende mucho de los casos.

Tabla 3. Comportamientos sanitarios en función del sexo

\begin{tabular}{|lllll|}
\hline $\begin{array}{l}\text { Sexo del } \\
\text { encuestado }\end{array}$ & $\begin{array}{l}\text { Número de consultas } \\
\text { realizadas a un } \\
\text { profesional de la salud en } \\
\text { las dos últimas semanas }\end{array}$ & $\begin{array}{l}\text { Tiempo de } \\
\text { espera en el } \\
\text { lugar de la } \\
\text { consulta }\end{array}$ & $\begin{array}{l}\text { Tiempo de } \\
\text { duración de la } \\
\text { consulta }\end{array}$ & $\begin{array}{l}\text { Satisfacción con la } \\
\text { consulta (puntuación } \\
\text { inversa) }\end{array}$ \\
\hline Hombre & 0,40 & 16,14 & 14,47 & 1,65 \\
Mujer & 0,59 & 19,25 & 16,03 & 1,55 \\
Total & 0,49 & 17,99 & 15,40 & 1,59 \\
\hline
\end{tabular}

Estos datos se traducen en una valoración positiva por parte de los vecinos y vecinas del barrio de su centro de salud. La mayoría de los encuestados (94,9\%) manifiestan encontrarse muy satisfechos con la atención recibida. Las mujeres, como principales usuarias de dichos servicios, constituyen el colectivo más satisfecho con dicha relación.

\subsection{Dinámicas de apoyo e integración comunitarias}

Los residentes en Casablanca manifiestan en la entrevista su confianza en la acción social de las organizaciones gubernamentales y no gubernamentales. De hecho, expresan que, en caso de necesidad, acudirían en busca de apoyo con más facilidad a asociaciones deportivas y culturales, grupos sociales y cívicos, parroquias, amas de casa, etc., que a servicios sociales, centros educativos o centros de salud.

Tabla 4. Análisis descriptivo del apoyo comunitario

\begin{tabular}{|l|l|l|l|l|}
\hline Estadísticos & Participación & Integración & $\begin{array}{l}\text { Sistemas } \\
\text { informales }\end{array}$ & $\begin{array}{l}\text { Sistemas } \\
\text { formales }\end{array}$ \\
\hline Media zaragozana & 16,69 & 14,87 & 28,00 & 12,00 \\
\hline Media valenciana & 16,23 & 16,90 & 30,78 & 13,51 \\
\hline
\end{tabular}

En anteriores investigaciones constatamos cómo las dinámicas de apoyo e integración comunitaria se relacionan directamente con comportamientos relativos a la salud 
(Gil-Lacruz, 20006). Las conductas de riesgo y la morbilidad de los encuestados afectan a su grado de participación efectiva. Así, padecer dolencias e incapacitaciones dificulta las conductas asociativas. A su vez, la integración y el apoyo percibido de los sistemas informales son predictores de una valoración positiva de los recursos comunitarios de carácter público. Esta interdependencia es importante en la valoración poblacional del acceso a los recursos sanitarios.

Una vez desarrollada una primera aproximación o diagnóstico del barrio, nos planteamos profundizar en los resultados obtenidos en estos cuestionarios priorizando la influencia de la variable sexo. Con este fin, hemos utilizado el modelo simple de comportamiento ANOVA de una sola variable de intervalo y una o varias variables nominales (Serra, 1994). Se ha elegido como nivel mínimo de significación estadístico $\alpha=$ 0,05 (Serret, 1995), sin pruebas posteriores, por tratarse de una variable dicotómica (Canovos, 1988).

\subsection{Comportamientos y recursos sanitarios}

Hombres y mujeres difieren en el motivo, en el lugar de la consulta y en la solución que los recursos sanitarios les aportan. Sin embargo, no se hallan diferencias estadísticamente significativas ni en los tiempos de espera y duración de la consulta, ni en el grado de satisfacción con que los vecinos y vecinas evalúan dicha interacción.

A fin de predecir la utilización de los recursos sanitarios se realizaron análisis de varianza con otras variables. Las consultas de los hombres menores de 29 años tienden a ser más largas que las de las personas mayores de 60, y es precisamente el colectivo juvenil el que se manifiesta encontrarse más a gusto con la atención recibida. Las personas con altos niveles de instrucción académica (universitarias) y que trabajaban en el momento de responder a la entrevista también comparten un mayor grado de satisfacción con dichas consultas.

En las zonas originarias y precarizadas del barrio, la percepción de los vecinos acerca de su estado de salud es comparativamente peor que en otras zonas del barrio. Pese a esto, los resultados referentes a los indicadores sanitarios no conllevan ni una mayor

\footnotetext{
6 En la monografía Salud y fuentes de apoyo social, publicada por el Centro de Investigaciones Sociológicas, se explicitan dichos resultados. El modelo de investigación e intervención basado en el apoyo comunitario es congruente con un modelo de salud social. Los estudios sobre apoyo comunitario, al demostrar la relación existente entre las interacciones sociales y el bienestar de las personas, se constituyen en una fuente de evidencia empírica de cómo los grupos de referencia son agentes de motivación e influencia tanto de la vulnerabilidad y las conductas de riesgo que las personas adoptan como de los comportamientos saludables, preventivos, terapéuticos y, en cualquier caso, de trascendencia positiva de uno mismo. En un sentido similar, el metaanálisis desarrollado por Schwarzer y Leppin (1991) ofreció resultados contundentes sobre la estrecha conexión entre morbilidad / mortalidad y apoyo (a partir de 80 estudios que constituyen un total de de 60.976 individuos repartidos en 110 muestras).
} 
utilización de las consultas sanitarias, ni diferencias significativas en cuanto a la duración y satisfacción.

Tabla 5. Análisis de varianza de indicadores sanitarios

\begin{tabular}{|l|l|l|l|l|l|}
\hline Indicadores sanitarios & $\begin{array}{l}\text { Suma de } \\
\text { cuadrados }\end{array}$ & F & Sig. & Hombre & Mujer \\
\hline Número de consultas & 9,171 & 6,328 & $\mathbf{0 , 0 1 2}$ & 0,40 & 0,58 \\
\hline Tiempo de espera previo a la consulta & 717,126 & 1,153 & 0,283 & 16,14 & 19,25 \\
\hline $\begin{array}{l}\text { Tiempo de duración promedio de la } \\
\text { consulta }\end{array}$ & 178,588 & 0,544 & 0,461 & 14,47 & 16,03 \\
\hline Grado de satisfacción atención recibida & 0,593 & 1,443 & 0,230 & 1,63 & 1,54 \\
\hline
\end{tabular}

\subsection{Procesos de integración, participación y valoración de los sistemas informales}

Respecto a los procesos de integración, participación en la comunidad de referencia y valoración de los sistemas informales, no se han hallado diferencias entre sexos. Sí que se aprecian, en cambio, tendencias diferenciales en la valoración del apoyo procedente de los sistemas formales, ya que las mujeres comparten una visión más positiva de los servicios sociales, centros educativos y centros de salud como posibles fuentes de ayuda en caso de necesidad.

A su vez, y tras el correspondiente análisis, observamos que esta confianza en los organismos gubernamentales se puede predecir mediante la medida en que la encuestada se identifica con el barrio y con una valoración igualmente optimista de los sistemas informales de apoyo (Gil-Lacruz et alii, 2000).

Asimismo, se aprecian percepciones diferenciales en cuanto a la identificación con el barrio. Los vecinos que residen en zonas recientes (Fuentes Claras) son los que menos se integran en la dinámica comunitaria. Estas diferencias coinciden con los años de asentamiento en el barrio, que, a su vez, influyen directamente en el sentimiento de pertenencia e identificación con la comunidad y en la valoración positiva de los recursos gubernamentales que se encuentran en Casablanca.

Tabla 6. Análisis de varianza de apoyo e integración

\begin{tabular}{|l|l|l|l|l|l|}
\hline Variables del cuestionario AC & $\begin{array}{l}\text { Suma de } \\
\text { cuadrados }\end{array}$ & F & Sig. & Hombre & Mujer \\
\hline Participación & 0,044 & 0,007 & 0,933 & 16,684 & 16,70 \\
\hline Integración & 8,619 & 2,267 & 0,132 & 14,970 & 14,79 \\
\hline
\end{tabular}




\begin{tabular}{|l|l|l|l|l|l|}
\hline Sistemas informales & 0,162 & 0,021 & 0,884 & 30,80 & 30,77 \\
\hline Sistemas formales & 26,870 & 11,031 & 0,000 & 13,35 & 13,67 \\
\hline
\end{tabular}

\section{Conclusiones}

En nuestro trabajo se observa cómo las diferencias en estratificación social (ej. ingresos, estudios, situación laboral, etc.) y condiciones de vida se traducen en un acceso desigual a los servicios sanitarios y generan diferencias de salud (Arias et alii, 1993). Las desigualdades de poder, prestigio, estatus, respeto y autoestima generan una mayor vulnerabilidad en las poblaciones que son y se perciben menos privilegiadas en su entorno social. Las diferencias en cuanto a sexo no hacen sino profundizar en estas tendencias.

Necesitamos marcos teóricos que nos ayuden a interpretar la influencia de las variables. Por ejemplo, el apoyo social se puede definir como una variable antecedente y/o intermedia en la explicación de la salud (Gil-Lacruz et alii, 2000; Rodríguez, 1995). Por el contrario, el aislamiento supone una mayor vulnerabilidad y un menor acceso a recursos de ayuda en casos de necesidad. Coincidimos con Pederson (1982) en que es preciso profundizar en la forma como el contexto inmediato (grupo familiar, vecindario), el microcontexto (las redes sociales de apoyo, valores de grupo) y el contexto global o macrocontexto (sistema político, económico, sanitario) interactúan modelando y construyendo la experiencia de salud-enfermedad e inducen distintos tipos de tratamiento relacionados con la salud. Esta declaración supone reivindicar investigaciones centradas en lo local, en un mundo en que la salud se enmarca en procesos globalizadores.

Las variables diana de las investigaciones comunitarias son, frecuentemente y en buena lógica, las variables diana de las intervenciones sociales. Por eso, para reducir las desigualdades de salud, no sólo debe implicarse el sector sanitario, facilitando el estudio y la igualdad de acceso a dichos servicios, sino que también se deben reorientar las políticas sociales dirigidas a reducir las desigualdades en las condiciones «locales» de vida.

Un indicador de vulnerabilidad social es precisamente el género. La inequidad social afecta a las mujeres en mayor medida que a los varones, en tanto que recorta sus oportunidades y las excluye de los beneficios del desarrollo. La inequidad de género se expresa en el grado de acceso y de control ejercido por las personas sobre los recursos básicos para la producción de la salud tanto a nivel intrafamiliar como a nivel público (alimentos, información, trabajo remunerado, seguridad social, etc.). En Casablanca, las vecinas acuden por lo general a los servicios sanitarios de titularidad pública y con- 
sultan con menos frecuencia los recursos médicos de los centros laborales y las mutualidades privadas (seguramente, porque su situación laboral no se lo permite) que los hombres.

Esta conducta diferencial se observa también en el hecho de que la mujer es, con frecuencia, el agente social que facilita el acceso al servicio sanitario público de su red social. Al definirse la identidad femenina como «cuidadora», debido a los valores de la ideología patriarcal predominante, las mujeres son percibidas como agentes de salud por su familia y comunidad.

La concentración de las políticas sociales en programas materno-infantiles y de planificación familiar tiende a visualizar a las mujeres sólo como reproductoras y responsables de la salud de los hijos y pierde de vista una perspectiva de salud integral (García et alii, 1986). ${ }^{7}$ El cambio de situación supone un proceso técnico y político, que requiere cambios en la cultura organizativa y en la manera de pensar, así como en los objetivos, las estructuras y la distribución de recursos de las agencias internacionales, gobiernos y organizaciones no gubernamentales (Ministerio de Sanidad y Consumo, 2003).

El objetivo final es la capacitación y empowerment (empoderamiento) de la población. Para ello, se requiere incidir en una metodología de educación para la salud que potencie el empoderamiento de las mujeres, creando las circunstancias que favorezcan su confianza, dignidad y capacidad de enfrentamiento colectivo. En definitiva, necesitamos un cambio de modelo interventor donde las nuevas consignas sean la prevención, la promoción y la equidad.

\section{Bibliografía}

Alonso J.; Antó J. M. (1986). Enquesta de salut de Barcelona. Barcelona: Ayuntamiento de Barcelona.

Arias, A.; Eebagliato, M.; Palumbo, M. A.; Bellver, R.; Ashton, J.M.; Colomer, C.; Costa, J.; Flynn, P.; Álvarez Dardet, C. (1993). «Desigualdades en salud en Barcelona y Valencia», Medicina Clínica, 100: 281-287.

7 La igualdad de género en el sistema de salud supondría la ausencia de discriminación basada en el sexo en cuanto a las oportunidades, la distribución de recursos o beneficios y el acceso a los servicios. La equidad de género significaría imparcialidad y justicia en la distribución de los beneficios, el poder, los recursos y las responsabilidades entre hombres y mujeres. Este concepto reconoce que las necesidades de las mujeres y los hombres son diferentes, y que las diferencias deben ser identificadas y tratadas para corregir el desequilibrio existente entre los sexos (Ministerio de Salud y Consumo, 2003). 
Armero, M. J; Frau, M. J;; Colomer, C. (1991). «Indicadores de salud en el medio urbano. Variaciones en función de la coherencia social de las áreas geográficas utilizadas». Gaceta Sanitaria, 22: 17-20.

Cais, J.; Castilla, E. J. (1995). «El sector sanitario. Síntesis del V Informe sociológico sobre la situación social en España», Documentación Social: 227-272.

Canovos, G. C. (1988). Probabilidad y estadística. Aplicaciones y métodos. Madrid: McGraw-Hill.

Clark, J. D. (1990). «Variation in Michigan Hospital use rates: de physician and hospital characteristics provide the explanations?», Soc Sci Med, 30:67-82.

De Miguel, J. M. (1997). La sociedad española 1996 -1997. Madridः Universidad Complutense.

Durán, M. A. (1983). Desigualdad social y enfermedad. Madrid: Tecnos.

Duro, J. C. (2001). Psicología y salud comunitaria durante la transición democrática. Madrid: Colegio Oficial de Psicólogos.

Esteban, M. L. (1996). «Relaciones entre feminismo y sistema médico científico», en Ortiz, T.; Becerra, G. (eds.). Mujeres de ciencia. Mujer, feminismo y ciencias naturales, experimentales y tecnológicas. Granada: Universidad de Granada.

Federación de Asociaciones de Vecinos (1981). Zaragoza, barrio a barrio: Casco Viejo, Casablanca. Zaragoza: Ayuntamiento de Zaragoza.

García, M. A.; García, M.; Ortega, F. (1996). Sociología de las mujeres españolas. Madrid: Editorial Complutense.

Gil-Lacruz, M. (2000). Salud y fuentes de apoyo social. Análisis de una comunidad. Madrid: Centro de Investigaciones Sociológicas.

Gil-Lacruz, A. I. (2004). Análisis económico del consumo de alcohol entre los jóvenes: capital bumano, capital social y bienestar. Tesis doctoral. Universidad de Zaragoza.

Gil-Lacruz, M.; Abadía, J.; Aragón, R.; García y Matute, S. (2000). Análisis psicosocial de recursos sanitarios. Zaragoza: Mira.

Gordon, E.; Golanty, E.; Browm, K. M. (1999). Health and Wellness. Sudbury; Jones and Bartlett Publishers.

Gracia, E*; Musitu, G. (1990). «Integración y participación en la comunidad: una conceptualización empírica del apoyo social comunitario», en Musitu, G.; BorJano, E.; Bueno, J. R. (eds.). Psicología comunitaria. Valenciaः Nau Llibres.

Hahn, D. B.; Payne, W. A. (1999). Focus on Health. Boston: WCB/McGrawHill.

Martínez, M. A.; Guillén, E. «Estado de Bienestar y servicios sociales: problemas, reacciones y medidas necesarias», en CASIldA BÉJAR, R.; TortosA, J. M. (eds.). Pros y contras del Estado de Bienestar. Madrid: Tecnos. 
Mateo Rivas, M. J*; García Ferrando, M. (1989). Estadística aplicada a las ciencias sociales. Madrid: UNED.

Ministerio de Sanidad y Consumo (2003). La equidad de género en la salud mediante el mainstreaming. Madrid: Centro de Publicaciones del Ministerio.

Moreno, L*; Pérez, M. (1992). Política social y Estado de Bienestar. Madrid: Ministerio de Asuntos Sociales.

Navarro, M. D. (1994). Encuestas de salud. Madrid: Centro de Investigaciones Sociológicas. Cuadernos Metodológicos, 11.

Olson, D. A.; Shultz, K. S. (1994). «Gender differences in the dimensionality of social support», J App Soc Psy, 24 (14): 1221-1232.

OMS (1986). Glosario de promoción de la salud. Ginebra: OMS.

OMS (1996). The Women's bealth and development programme. Famile and reproductive Health. Ginebra: OMS.

Pederson, D. (1982). «Estilos de vida», en Mazzafero, V. E. (ed.). Medicina en salud pública. Buenos Aires: Ateneo.

Rodríguez, J. (1995). Psicología social de la salud. Madrid: Síntesis.

Schwarzer, R.; Leppin, A. (1991). «Social support and health: A theoretical and empirical overview», Journal of Social Personal Relationships, 8: 99-127.

Serra, R. (1994). Análisis estadístico multivariable: teoría y ejercicios. Madridः Paraninfo.

Serret, J. (1995). Manual de estadistica universitaria inductiva. Madrid: ESIC.

YAGo, T. (2002). Inmigrantes y además mujeres. La inmigración una realidad en España. Zaragoza: Seminario de Investigación para la Paz. Colección «Actas» núm 57.

\section{Anexo. AC-90 \\ (Gracia y Musitu, Apoyo Comunitario, 1990)}

Recuerde que toda la información es anónima y no le compromete a nada.

\begin{tabular}{|c|c|c|c|c|c|}
\hline $\begin{array}{l}\text { A continuación le voy a leer una serie de frases. Dígame cómo se } \\
\text { sitúa con respecto a ellas. }\end{array}$ & 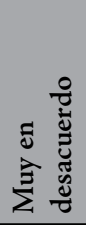 & 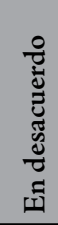 & 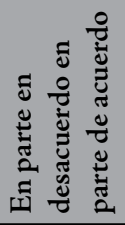 & 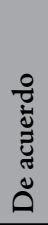 & 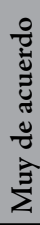 \\
\hline 1. Me encuentro a disgusto dentro de mi barrio o comunidad & 1 & 2 & 3 & 4 & 5 \\
\hline 2. Me siento identificado con mi comunidad/barrio & 1 & 2 & 3 & 4 & 5 \\
\hline 3. Mis opiniones son bien recibidas en mi barrio o comunidad & 1 & 2 & 3 & 4 & 5 \\
\hline
\end{tabular}




\begin{tabular}{|c|c|c|c|c|c|}
\hline 4. Muy pocas personas de mi comunidad saben quién soy yo & 1 & 2 & 3 & 4 & 5 \\
\hline 5. Siento el barrio como algo mío & 1 & 2 & 3 & 4 & 5 \\
\hline $\begin{array}{l}\text { 6. Colaboro en las organizaciones y asociaciones de mi } \\
\text { comunidad }\end{array}$ & 1 & 2 & 3 & 4 & 5 \\
\hline 7. Participo en actividades sociales de mi barrio o comunidad & 1 & 2 & 3 & 4 & 5 \\
\hline $\begin{array}{l}\text { 8. Participo en algún grupo social o cívico (asociaciones de } \\
\text { vecinos, amas de casa, etc.) }\end{array}$ & 1 & 2 & 3 & 4 & 5 \\
\hline $\begin{array}{l}\text { 9. No participo en las reivindicaciones sociales que se llevan a } \\
\text { cabo en mi barrio/comunidad }\end{array}$ & 1 & 2 & 3 & 4 & 5 \\
\hline $\begin{array}{l}\text { 10. Acudo a las llamadas de apoyo que se hacen dentro de mi } \\
\text { comunidad }\end{array}$ & 1 & 2 & 3 & 4 & 5 \\
\hline $\begin{array}{l}\text { 11. No participo en las actividades sociorecreativas de mi } \\
\text { comunidad }\end{array}$ & 1 & 2 & 3 & 4 & 5 \\
\hline $\begin{array}{l}\text { Como usted sabe, asociaciones deportivas o culturales, grupos } \\
\text { sociales o cívicos (asociaciones de vecinos, amas de casa, } \\
\text { de consumidores, comisión de fiestas, etc.), la parroquia, } \\
\text { agrupaciones políticas o sindicales, etc., son organizaciones } \\
\text { donde se desarrolla una parte importante de la vida social de las } \\
\text { personas. En estas organizaciones: }\end{array}$ & 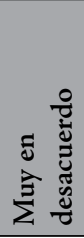 & 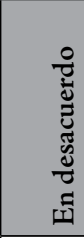 & 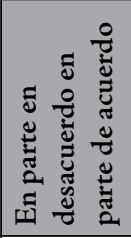 & 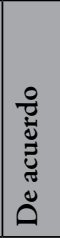 & 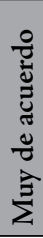 \\
\hline $\begin{array}{l}\text { 12. Podría encontrar personas que me ayudaran a resolver mis } \\
\text { problemas }\end{array}$ & 1 & 2 & 3 & 4 & 5 \\
\hline 13. No encontraría comprensión y apoyo & 1 & 2 & 3 & 4 & 5 \\
\hline $\begin{array}{l}\text { 14. Podría encontrar a personas que me ayudaran a sentirme } \\
\text { mejor }\end{array}$ & 1 & 2 & 3 & 4 & 5 \\
\hline 15. No pediría consejo & 1 & 2 & 3 & 4 & 5 \\
\hline 16. No podría compartir mis problemas & 1 & 2 & 3 & 4 & 5 \\
\hline $\begin{array}{l}\text { 17. Encontraría a alguien que me escuchara cuando estoy } \\
\text { decaído }\end{array}$ & 1 & 2 & 3 & 4 & 5 \\
\hline 18. Encontraría una fuente de satisfacción para mí & 1 & 2 & 3 & 4 & 5 \\
\hline 19. Lograría animarme y mejorar mi estado de ánimo & 1 & 2 & 3 & 4 & 5 \\
\hline $\begin{array}{l}\text { 20. Me relajaría y olvidaría con facilidad mis problemas } \\
\text { cotidianos }\end{array}$ & 1 & 2 & 3 & 4 & 5 \\
\hline 21. No solicitaría ayuda & 1 & 2 & 3 & 4 & 5 \\
\hline $\begin{array}{l}\text { Servicios sociales, centros educativos y centros de salud son } \\
\text { organizaciones y servicios que la Comunidad pone a disposición } \\
\text { de sus miembros. }\end{array}$ & 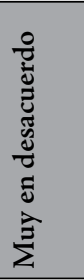 & 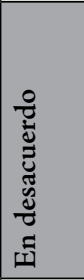 & 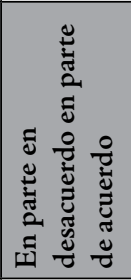 & 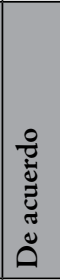 & 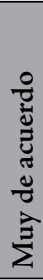 \\
\hline $\begin{array}{l}\text { 22. Si tuviera problemas (personales, familiares, etc.), en estas } \\
\text { organizaciones podría encontrar personas que me ayudarían a } \\
\text { resolverlos }\end{array}$ & 1 & 2 & 3 & 4 & 5 \\
\hline 23. Estos servicios no me inspiran la suficiente confianza & 1 & 2 & 3 & 4 & 5 \\
\hline $\begin{array}{l}\text { 24. Estas organizaciones y servicios son una importante fuente } \\
\text { de apoyo }\end{array}$ & 1 & 2 & 3 & 4 & 5 \\
\hline 25. En caso de necesidad acudiría a estas organizaciones & 1 & 2 & 3 & 4 & 5 \\
\hline
\end{tabular}

